\title{
Ewing's Sarcoma of mandible in a pediatric patient: A case report
}

\author{
Satish Verma ${ }^{1 *}$, Guru Prasad R ${ }^{2}$, Neeta Sharma ${ }^{3}$, Narottam Ghejta ${ }^{4}$ \\ ${ }^{1}$ Post Graduate Student, ${ }^{2}$ Professor and HOD, ${ }^{3}$ Professor, ${ }^{4}$ Assistant Professor, ${ }^{1-3}$ Dept. of Oral Medicine, ${ }^{4}$ Dept. of Maxillofacial Surgery, \\ Diagnosis and Radiology, HP GDC, Shimla, Himachal Pradesh, India
}

*Corresponding Author: Satish Verma

Email: dentsatish74@gmail.com

\begin{abstract}
Ewing's sarcoma is a small round-cell malignant tumor of bones, primarily affecting children and young adults. It might be neuroectodermally derived with various degrees of differentiation of the primitive neural tissues. It has retained the most unfavourable prognosis of all primary musculoskeletal tumors, with long-term survival being less than $10 \%$. The development of multi-disciplinary therapy with chemotherapy, irradiation and surgery, the survival rates are increased to greater than $50 \%$. The pathognomonic translocations involving the EWS gene on chromosome 22 and an ETS-type gene, which is the Fli1 gene on chromosome 11, are implicated in more than 95\% of Ewing's sarcomas. This paper reports a rare case of ES of mandible in 8years old male patient. The patient was managed with multiagent chemotherapy, followed by surgical resection.
\end{abstract}

Keywords: Ewing's sarcoma, Mandible, Malignant tumor, Surgery, Chemotherapy.

\section{Introduction}

Ewing's sarcoma (ES) is a rare malignant small round cell tumour that primarily affects the skeletal system. It accounts for 4 to $10 \%$ of all types of bone cancer, with long bones and pelvis being the most common locations. ${ }^{1}$ It affects mainly adolescents and young adults. It rarely involves age group below 5 and above 30 yrs. ${ }^{1,2}$ Clinically, this tumour has an aggressive behaviour with rapid growth and high probability of micrometastasis. ${ }^{3}$ Radiographic investigations exhibit onion skin-like appearance, indicative of infiltrative destruction of the affected bone. Computed tomography (CT) is useful for depicting extraskeletal soft tissue masses, destruction of bone cortex and pulmonary metastasis. MRI exhibits low signal intensity on T1-weighted images and high signal intensity on T2-weighted images and appears as large extra skeletal soft tissue masses derived from bone. ${ }^{1,2}$ It displays high 18F-fluorodeoxy glucose uptake on 18F-FDGpositron emission tomography. Histologically, the tumour consists of small round cells with regular round nuclei containing finely dispersed chromatin and inconspicuous nucleoli and a narrow rim of clear or pale cytoplasm. ${ }^{4}$ Accurate diagnosis of ES is crucial for the most appropriate management of the patients. Adequate clinical information and recognition of morphological, immunocytochemical and ultrastructural features of ES are required for its differential diagnosis from other small round cell tumours of childhood. ${ }^{2}$ Here we present the case report of 8yrs old male patient presented with clinical, radiographic and histopathological features characteristic of ES, who was managed with chemotherapy, followed by surgical intervention.

\section{Case Report}

An eight years old male patient reported to Department of Oral Medicine and Radiology of our institute with a chief complaint of swelling with occasional mild pain over left mid region of lower jaw since three months. Medical and family histories were non contributory. Past dental history revealed extraction of a carious tooth 75, four months ago. Extraoral examination revealed facial asymmetry with a diffuse, hard, non tender swelling present over left lower $1 / 3^{\text {rd }}$ region of face, extending to left ala-tragus line superiorly and lower border of the mandible inferiorly, anteriorly to left angle of mouth and posteriorly $2 \mathrm{~cm}$ anterior to the left angle of the mandible (Fig. 1a). Overlying skin was stretched and shiny. Intraorally, a well defined swelling was found over labial aspect of left mandibular alveolar ridge w.r.t. premolar and molar region measuring $2.5 \mathrm{~cm}$ mesiodistally and $1.5 \mathrm{~cm}$ labio-lingually with blanched pinkish white mucosal covering and diffuse borders (Fig 1b) and slight lingual expansion of the jaw was noticed. Bony crepitus was present over mid portion of the swelling and left mandibular buccal vestibule was obliterated due to extension of expansile bony growth. A provisional diagnosis of Benign bony tumor affecting mandible was made with differential diagnosis being Residual cyst, Dentigerous cyst, Garre's osteomyelitis, Ameloblastoma, Osteosarcoma, Chondrosarcoma, Ewing's sarcoma and Fibrosarcoma etc. The radiographic investigations including intraoral periapical radiograph wrt 73, 74, 75 region (Fig. 1c), mandibular occlusal radiograph (Fig 1d), OPG (Fig. 2), contrast enhanced computed tomography (CECT) of jaw, MRI, PA view Chest, PA view femur and scapula was done. A well defined multilocular osteolytic radiolucency was seen involving body of the mandible with "sun burst periosteal reaction" and displaced anterior tooth buds with well defined septa extending anteroposteriorily from 73 to distal aspect of 36 tooth with vertical extension from alveolar ridge superiorly to inferior border of mandible inferiorly (Fig. 2,3). Unerupted 33, 34 and 35 were displaced inferiorly and resorption of roots w.r.t. 74 and 36 was observed. CECT revealed a lytic expansile lesion involving body of mandible with spiculated reaction giving sun burst appearance. There was breach in anterior as well posterior aspect of labial surface of alveolar cortex extending in left submandibular and submental region (Fig 3a). MRI mandible scan shows heterogeneously enhancing erosion and 
destruction mass in relation to mandible on left side with its extension into floor of mouth, muscles and cheek (Fig. 3b).

ESR level, RDW and segmented neutrophils levels were raised with low hematocrit. Histopathological investigations showed multiple fragments of tumor tissues revealing sheets of tumor cells with hypochromatic round nuclei, eosinophilic cytoplasm and occasional mitotic figures with rosette formation with fibrillary center (Fig 4). Extensive area of tumor necrosis was seen, suggestive of "malignant small round cell tumor." On the basis of investigations, a final diagnosis of Ewing Sarcoma was made.

Patient was advised treatment based on multidisciplinary approach, including chemotherapy, followed by surgery.
Chemotherapy was advised for control of micrometastasis, that consisted of Multiagent therapy including vincristine, Dactinomycine, Cyclophosphamide and Doxorubicine. Surgery was planned for local control that included segmental resection of pathology bearing segment of the mandible (Fig. 5). After surgery, patient was advised to undergo chemotherapy. Regular follow-up every 3 months for the first year, every 6 months for the second year, and then yearly thereafter was advised to patient. Patient reported follow up regularly. After two years of follow up, CECT and clinical examination revealed no recurrence or metastasis. (Fig. 6).

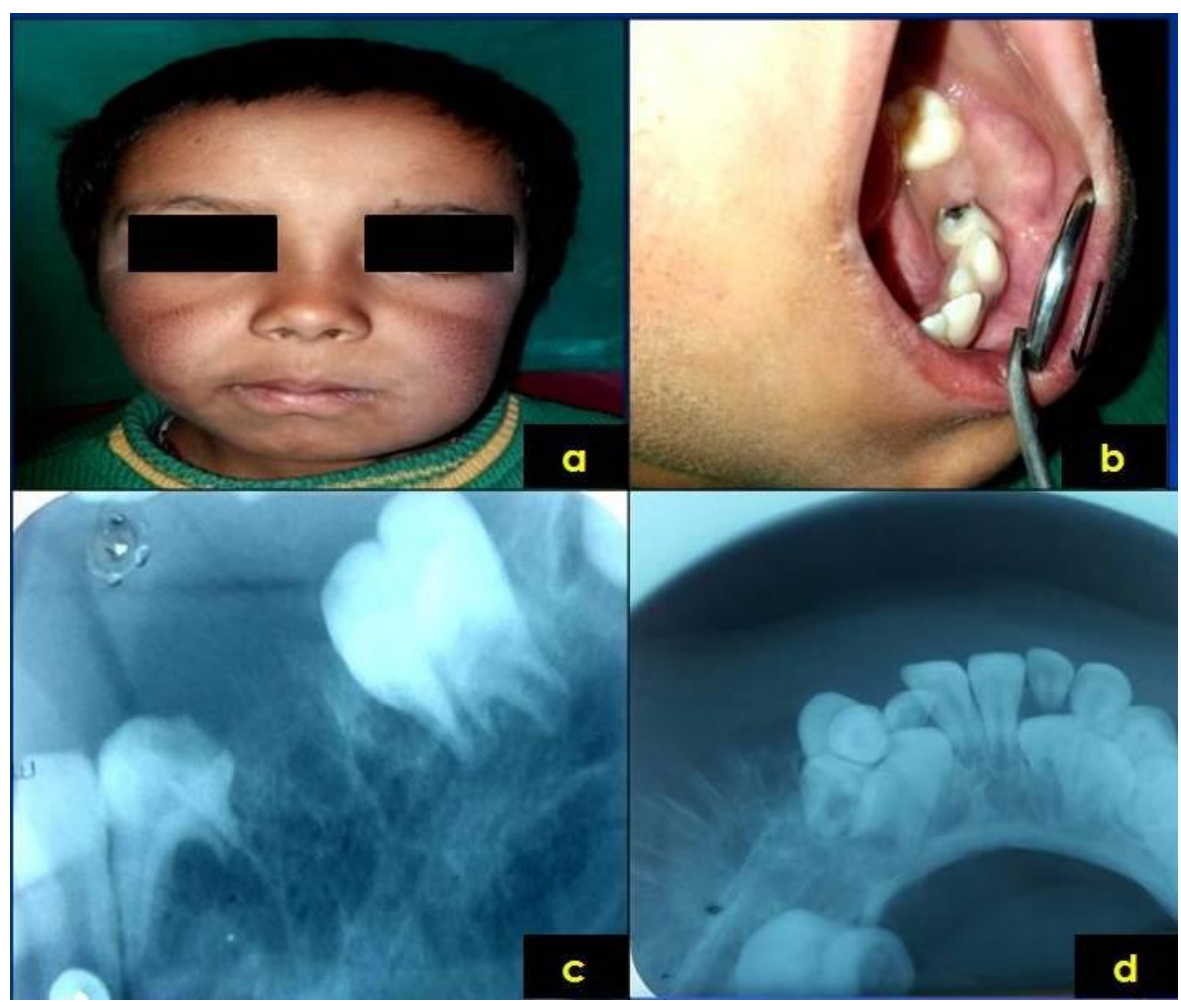

Fig. 1:a) Extraoral photograph showing facial asymmetry; b): A well defined swelling observed intraorally; c): Intraoral periapical radiograph; d): Mandibular occlusal radiograph showing multilocular osteolytic radiolucency

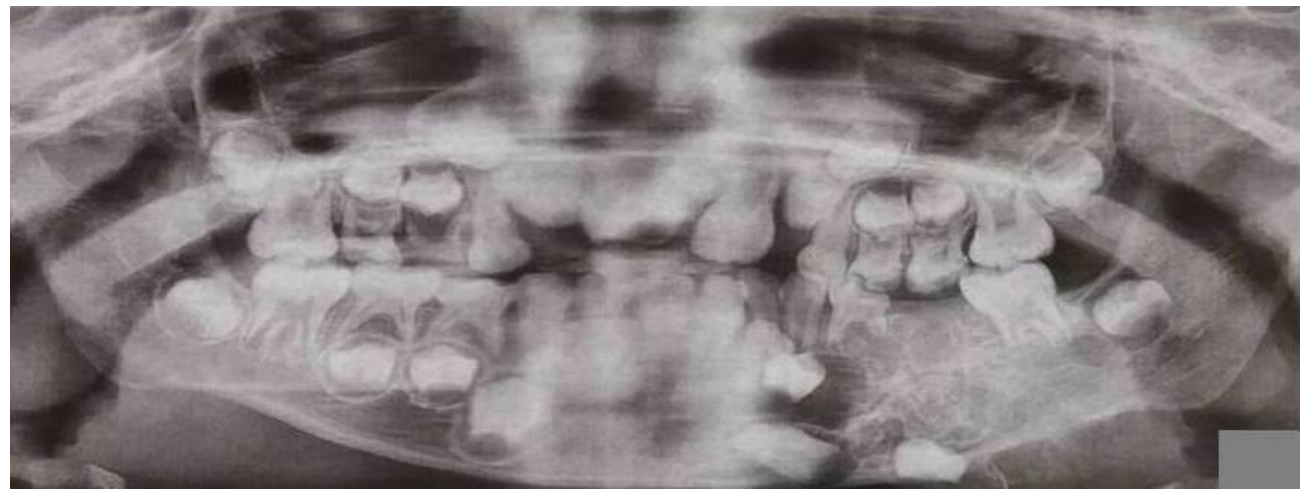

Fig. 2: OPG showing a "sun burst periosteal reaction" well defined septa extending antero-posteriorily from 73 to distal aspect of 36 


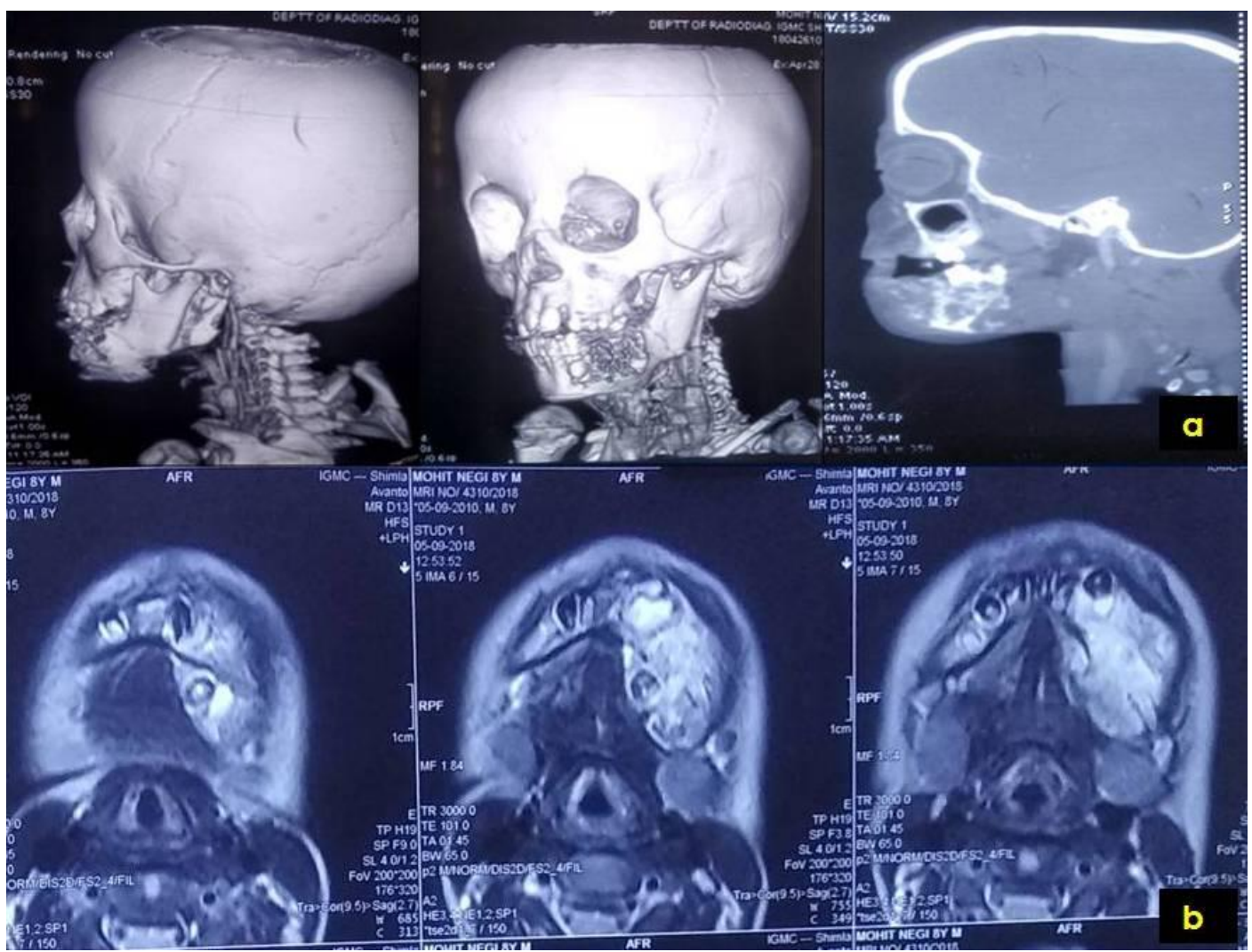

Fig. 3: a): CECT revealing a lytic expansile lesion involving body of mandible with spiculated reaction giving sun burst appearance; b): MRI mandible scan showing heterogeneously enhancing erosion and destruction mass in relation to mandible

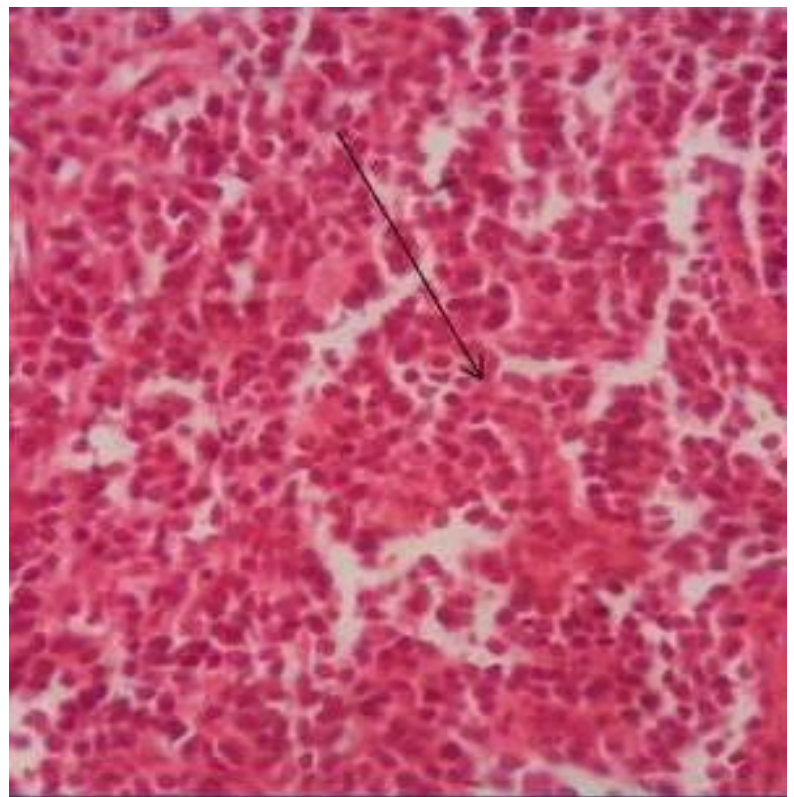

Fig. 4: Histopathological investigations showing rosette formation with fibrillary center 


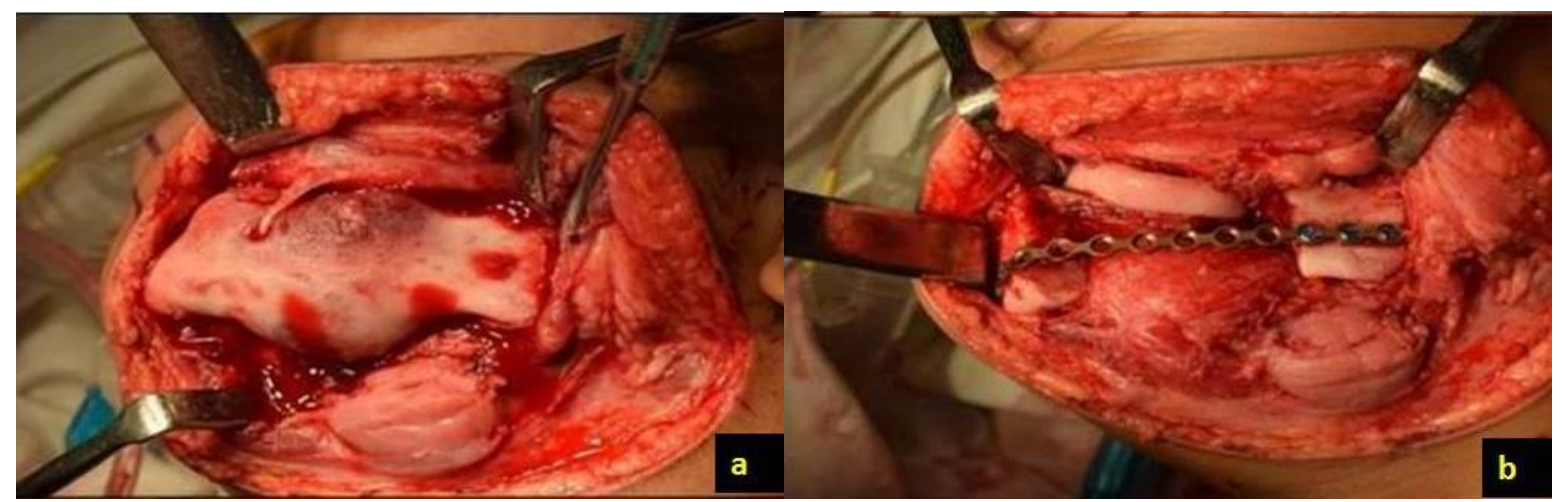

Fig. 5: Segmental resection of pathology bearing segment of the mandible

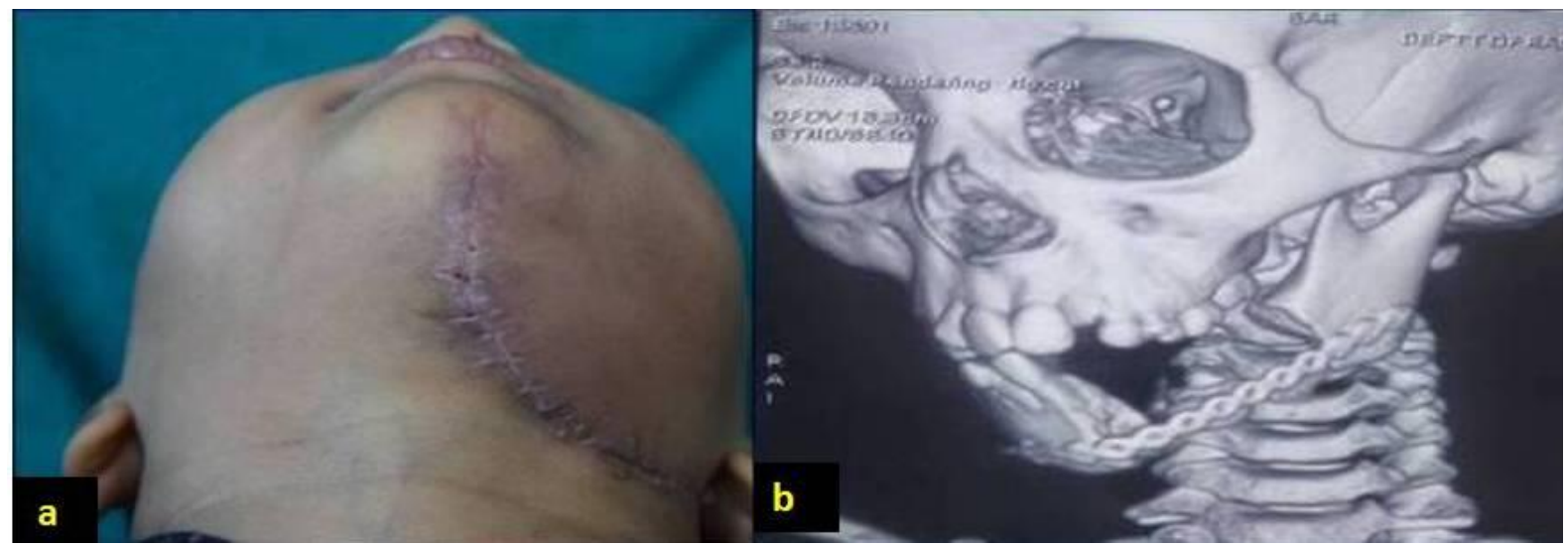

Fig. 6: Extraoral photograph and CECT revealing patient with no evidence of recurrence or metastasis after 2 years of follow up

\section{Discussion}

ES was first described by James Ewing in 1921 as a round cell neoplasm called 'diffuse endothelioma of bone'. ${ }^{5-6} \mathrm{ES}$ is related to the primitive neuroectodermal tumor (PNET), sharing a common karyotype translocation $t(11 ; 12)$ (q24;q12) in approximately $90 \%$ of these tumors. ${ }^{6-7} \mathrm{ES}$ affecting jaws is uncommon among Indian population. Potdar in 1970 first reported nine cases of ES involving jaws. ${ }^{6} \mathrm{ES}$ is a rare disease, making up only $1 \%$ to $4 \%$ in head and neck region, with more incidences in mandible $2: 1$ than maxilla. ${ }^{3}$ It is prevalent in posterior region as compared to anterior region, involving patients younger than 30 years with male predominance. ${ }^{3}$ In present case, patient was 8 years old with lesion in left mandibular posterior region.

ES presents with clinical symptoms that are nonspecific like pain, loosening of teeth, rapidly growing swelling of affected area, otitis media, paresthesia, etc. Systemic symptoms being fever, lymphadenopathy, weight loss, anemia, albuminuria are also observed. ${ }^{8}$ Radiographically, it presents as an ill-defined osteolytic lesion with displacement of unerupted tooth follicles. The characteristic 'sun-burst' or laminar periosteal "onion skin" reaction is a common radiological feature of ES involving long bones, but is rarely seen in jaws. ${ }^{7-9}$ In the present case, the radiographic finding was an ill-defined osteolytic lesion associated with sun-ray spicules of periosteal bone. The radiographic findings were confirmed with CBCT scan and MRI in present case.
Histopathologically, ES is composed of small round anaplastic cells with medium-sized, round to oval nuclei, small nucleoli and scanty peripheral ring of cytoplasm. ${ }^{6,10}$ Definitive diagnosis of ES depends on histology and genetic confirmation. ES should be differenciated from other "small round cell tumours of childhood" like rhabdomyosarcoma, lymphoma, ESFT etc. ${ }^{11}$

ES has poor prognosis because of hematogenous spread and lung metastases occur rapidly. ${ }^{2}$ Overall survival at 5 years was found to be $53 \%,{ }^{7}$ which highlights its potential aggressiveness and need for clinical vigilance with appropriate follow-up care. The poor prognostic factors of ES include patient age $>17$ yrs, large tumor size $>8 \mathrm{~cm}$, metastasis observed, pelvic and sacral tumors, extra-osseous soft tissue extension, post radiation medullary tumors, systemic symptoms, high erythrocyte sedimentation rate, elevated serum lactate dehydrogenase levels and thrombocytosis. ${ }^{2,7}$ However, tumours in jaws have a better prognosis than those in long bones. ${ }^{6}$

Combined therapy including surgery, radiotherapy and chemotherapy is the best approach for ES. ${ }^{3}$ Multidisciplinary treatment protocols have dramatically improved the 5-year survival rate of patients from 16 to $75 \% .^{6-9}$ Radiotherapy can treat non resectable primaries and chemotherapy can suppress micrometastasis thus reducing tumour load before surgery. In our case, the patient underwent evaluation by a multidisciplinary tumor board that believed that the patient would have a good response to chemotherapy and could be 
treated with surgery before radiation therapy. Although radiation therapy was not used in our case, its use should be considered. Despite substantial associated morbidity, including visual disturbances, pituitary dysfunction, and xerostomia, due to favorable surgical margins in our case, radiation therapy was ultimately avoided and chemotherapy was given postoperatively as well. Previous studies supported the use of radiotherapy along with chemotherapy as an initial management option for large tumors $(>10 \mathrm{~cm}){ }^{7,8,10}$

After resection of Ewing's sarcomas, large bone defects should be reconstructed to restore the function of affected limbs. The main options for reconstruction include autogenous bone grafts, allogeneic bone grafts and endoprosthesis. ${ }^{7,11}$ Follow-up plan depends on the aggressiveness of tumor and clinician limitations.

\section{Conclusion}

ES is a rare pathology, a highly lethal disease with poor prognosis when following recurrence. ${ }^{4}$ Advancement in standard treatment approaches have not been encouraging the survival rates for metastatic and recurrent cases, as 5-year survival rates have recently been described as less than $25 \%$. A comprehensive management including surgery, chemotherapy and radiation treatments are reaching their efficacy and toxicity limits. Thus, it is essential to improve the efficacy and utilization of targeted molecular therapies that may improve clinical management and survival for patients with this disease.

\section{Source of funding}

None.

\section{Conflict of interest}

None.

\section{References}

1. Ozaki T. Diagnosis and treatment of Ewing sarcoma of the bone: a review article. J Orthop Sci 2015;20(2):250-63.

2. Burchill SA. Ewing's sarcoma: diagnostic, prognostic, and therapeutic implications of molecular abnormalities. J Clin Pathol 2003;56:96-102.

3. Sripathi Rao BH, Rai G, Hassan S, Nadaf A. Ewing's sarcoma of the mandible. Natl J Maxillofac Surg 2011;2:184-8.

4. Ross KA, Smyth NA, Murawski CD, Kennedy JG. The biology of Ewing sarcoma. ISRN Oncol 2013 Article ID 759725:1-7.

5. Krishna KBB, Thomas V, Kattoor J, Kusumakumari P. A radiological review of Ewing's sarcoma of mandible: A case report with one year follow-up. Int J Clin Pedia Dent 2013;6(2):109-14.

6. Mukherjee A, Ray JG, Bhattacharya S, Deb T. Ewing's sarcoma of mandible: A case report and review of Indian literature. Contemp Clin Dent 2012;3:494-8.

7. Iwamoto Y. Diagnosis and treatment of Ewing's sarcoma. Jpn J Clin Oncol 2007;37(2)79-89.

8. Dadhe DP, Garde JB, Sambhus MB. Ewing's sarcoma in maxilla in an adolescent boy. J Int Clin Dent Res Organ 2010;3:153-6.

9. Gupta S, Gupta OP, Mehrotra S, Mehrotra D. Ewing sarcoma of the maxilla: A rare presentation. Quintessence Int 2009;40:135-40.
10. Rao BH, Rai G, Hassan S, Nadaf A. Ewing's sarcoma of the mandible. Natl J Maxillofac Surg 2011;2:184-8.

11. Potdar G. Ewing's tumors of the jaws. Oral Surg Oral Med Oral Pathol 1970;39:505-12.

How to cite this article: Verma S, Prasad GR, Sharma N, Ghejta N. Ewing's Sarcoma of mandible in a pediatric patient: A case report. J Oral Med, Oral Surg, Oral Pathol, Oral Radiol 2019;5(3):80-4. 